\title{
Autocrine VEGF and IL-8 Promote Migration via Src/Vav2/Rac1/PAK1 Signaling in Human Umbilical Vein Endothelial Cells
}

\author{
Li Jua Zhiwen Zhou $^{a}$ Bo Jiang ${ }^{a}$ Yue Lou ${ }^{a}$ Xirong Guo b,c \\ aChildren's Hospital of Nanjing Medical University, Nanjing, bepartment of Pediatrics, Nanjing \\ Maternity and Child Health Care Hospital of Nanjing Medical University, Nanjing, China \\ Institute of Pediatrics, Nanjing Medical University, Nanjing, Jiangsu, China
}

\section{Key Words}

VEGF $・$ IL-8 $\cdot$ Src $・$ Rac1 $・$ PAK1 $・$ Endothelial cells

\begin{abstract}
Background/Aims: Pro-angiogenic factors VEGF and IL-8 play a major role in modulating the migratory potential of endothelial cells. The goal of this study was to investigate the effect of autocrine VEGF and IL-8 in the form of self-conditioned medium (CM) on human umbilical vein endothelial cells (HUVECs). Methods: Enzyme-linked immunosorbent assay (ELISA) examined the automatic secretion of VEGF and IL-8 protein by HUVECs. Western blot, small interfering RNA (siRNA), pulldown and Transwell assays were used to explore the role and the mechanism of autocrine VEGF and IL-8 in migration of HUVECs. Results: Neutralizing VEGF and IL-8 in CM significantly abrogated CM-induced migration of HUVECs. Autocrine VEGF and IL-8 increased Src phosphorylation, Rac1 activity and PAK1 phosphorylation in a time dependent manner. Additionally, blocking Rac1 activity with Rac1 siRNA largely abolished autocrine VEGF and IL-8-induced cell migration. Vav2 siRNA suppressed autocrine VEGF and IL-8-induced Rac1 activation and cell migration. Furthermore, blocking Src signaling with PP2, a specific inhibitor for Src, markedly prevented autocrine VEGF and IL-8-induced Vav2 and Rac1 activation as well as consequently cell migration. PAK1 siRNA also significantly abolished autocrine VEGF and IL-8-induced cell migration. Conclusions: We demonstrated for the first time that autocrine VEGF and IL-8 promoted endothelial cell migration via the Src/Vav2/Rac1/PAK1 signaling pathway. This finding reveals the molecular mechanism in the increase of endothelial cell migration induced by autocrine growth factors and cytokines, which is expected to provide a novel therapeutic target in vascular diseases.
\end{abstract}

Xirong Guo, M.D, PhD. and Yue Lou, M.D, PhD.

KARGER
Department of Pediatrics, Nanjing Maternity and Child Health Care Hospital of Nanjing Medical University, 123rd Tianfei Street, Mochou Road, Nanjing 210004 (China) and Department of Pediatric Orthopaedics, Children's Hospital of Nanjing Medical University No.72 Guangzhou Road, Nanjing 210008 (China)

E-Mail xrguo@njmu.edu.cn / louyuenjmu@126.com 


\section{Cellular Physiology Cell Physiol Biochem 2017;41:1346-1359 \\ and Biochemistry Published onIIne: IVarch 09, $2017 \quad \begin{aligned} & \text { DOI: 10.1159/000465389 } 2017 \text { The Author(s). Published by S. Karger AG, Basel } \\ & \text { www.karger.com/cpb }\end{aligned}$}

Ju et al:: Autocrine VEGF and IL-8 Promote Migration of Endothelial Cells

\section{Introduction}

Angiogenesis is the formation of new blood vessels that sprouting from the pre-existing primitive vessels and occurs during normal development and in some disease states [1-3]. Migration of endothelial cells into the extracellular matrix towards the angiogenic factors plays a central role in angiogenesis [2].Of the growth factors involved in angiogenesis, vascular endothelial growth factor (VEGF) acts as a key regulator of endothelial cell migration through its tyrosine kinase receptors [4-7]. Cytokines, such as interleukin-8 (IL8), have also been recognized as angiogenic factors [8-11]. IL-8 could activate the vascular endothelial cells and induce migration behaviors [8]. VEGF and IL-8 secreted by cancer cells act as chemoattractants in promoting motility of endothelial cell during tumor angiogenesis [12-14]. It is noteworthy that VEGF and IL-8 are secreted not only by tumor cells, but also by endothelial cells, thereby enhancing endothelial cell migration and angiogenesis $[15,16]$ . However, the molecular mechanisms underlying the effect of autocrine VEGF and IL-8 on endothelial cell migration are not completely understood to date.

Rho GTPases transduce signals from extracellular stimuli to cause changes in cell behavior $[17,18]$. Of these GTPases, active Rac1 has been shown to stimulate cell migration by inducing lamellipodia formation $[19,20]$. It has been found that VEGF induce endothelial cell migration by activating Rac1 [21]. There is evidence showing that Rac1 is involved in IL-8 induced endothelial cell migration [22]. Like other Rho GTPases, Rac1 acts as a molecular switch that cycles from an inactive GDP bound state to an active GTP-bound state [23]. Rac1 is activated by guanine nucleotide exchange factors (GEFs) that promote the exchange of GDP to GTP [24]. There are 2 distinct families of Rho GEFs with 11 members in human [23]. They function upstream of Rac1 and promote Rac1-dependent cell migration [25, 26]. However, despite the abundance and importance of these GEFs in the spatio-temporal control of Rac1 activation, little is known about their implication during autocrine growth factors and cytokines induced endothelial cell migration.

The non-receptor tyrosine kinase Src regulates a number of signaling pathways that are involved in survival, adhesion, proliferation, invasion and migration [27, 28]. It has been shown that Src plays a critical role in regulating Rho GTPases such as Rac1, and one of the major functions of Src is to modulate the actin cytoskeleton that controls cell migration. A recent study reported that IL-8 up-regulated motility activity through Src signaling in human gastric cancer cells [29]. Src signaling has also been shown to be involved in VEGF-induced angiogenesis [30]. Hence, it will be interesting to clarify whether Src signaling may also be involved in autocrine VEGF and IL-8-induced endothelial cell migration.

P21-activated kinase 1 (PAK1), a direct downstream effector molecular of Rac1 and Cdc42, plays important roles in regulating cell motility, signal transduction, cell death and survival $[31,32]$. It has been reported that PAK1 activity was required for endothelial cell migration and permeability, and PAK1 inhibition by autoinhibitory domain of PAK1 also suppressed angiogenesis [33, 34]. In addition, Src has been identified as a potent modulator of extracellular signal-regulated PAK1 activity [35].Recent studies have suggested that PAK1 can be activated by growth factors and cytokines such as VEGF and IL-8 [34].Thus, it is worthwhile to explore whether the PAK1 signaling pathway is involved in autocrine VEGF and IL-8-induced endothelial cell migration. In the present study, we investigated the signaling mechanisms underlying the effect of autocrine VEGF and IL-8 on endothelial cell migration. The Src/Vav2/Rac1/PAK1 signaling pathway for autocrine VEGF and IL-8stimulated endothelial cell migration is identified.

\section{Materials and Methods}

Cell culture and collection of conditioned media

Human umbilical vein endothelial cells (HUVECs) were obtained from the Chinese Academy of Sciences Cell Bank (Shanghai, China) and grown in endothelial cell growth medium according to manufacturer's instructions at $37^{\circ} \mathrm{C}$ and $5 \% \mathrm{CO}_{2}$. COS-7 cells were grown in Dulbecco's modified Eagles medium (Sigma, 


\section{Cellular Physiology Cell Physiol Biochem 2017;41:1346-1359



Ju et al:: Autocrine VEGF and IL-8 Promote Migration of Endothelial Cells

St. Louis, MO) containing 10\% fetal bovine serum (Invitrogen, Carlsbad, CA) and antibiotics (100 units/ $\mathrm{ml}$ penicillin and $100 \mu \mathrm{g} / \mathrm{ml}$ streptomycin; Invitrogen). Conditioned medium (CM) was collected in sterile conditions followed by centrifugation at $1500 \times \mathrm{g}$ for $10 \mathrm{~min}$, and then stored at $-80^{\circ} \mathrm{C}$ until use. Unless otherwise mentioned, CM in this study is a self conditioned medium collected from the culture medium of HUVECs. Complete medium alone without cells was incubated under the same experimental conditions and referred as plain medium (PM) that served as control.

\section{Cell migration assay}

Cell migration was assessed in amodified Boyden chamber (Costar), in which two chambers were separated by a polycarbonate membrane (pore diameter, $8.0 \mu \mathrm{m}$ ). HUVECs were grown to subconfluence in tissue culture plates and then detached; thereafter, they were centrifuged and rendered into single cell suspensions in serum-free culture medium supplemented with $5 \mu \mathrm{g} / \mathrm{mL}$ BSA. The suspensions containing $2 \times 10^{4}$ cells were added to wells with a membrane placed in the bottom. The cells were allowed to migrate for the indicated periods of time at $37^{\circ} \mathrm{C}$ in this assay. Thereafter, the medium was discarded, stationary cells were removed with a cotton-tipped applicator and the membranes were cut out of the chamber and stained with $0.5 \%$ crystal violet. The response was evaluated in a light microscope by counting the number of cells that had migrated into the membrane.

Small interfering RNA (siRNA)

For gene knockdown, siRNA duplexes specific for Rac1 (On-Target Plus: $5^{\prime}$-CAC UCC CAU CAU CCU AGU GdT dT - $3^{\prime}, 5^{\prime}$-CAC CGA GCA CUG AAC UUU GdT dT $-3^{\prime}$ and $5^{\prime}$-CUG GAG GCC AAG AUA CUU CdT dT-3'), Vav2 (5'- GGA ACA GCG AGC UGU UUG AdT dT-3', 5' - UAC CAC CUG CUC UUG AAG GdT dT-3'and 5'-GGA GAU CAU CGA GCU GCU GdT dT -3') and PAK1 (On-Target Plus: 5'-UGA AAA CCC UCU GAG AGC CdT dT-3', 5'- GAA AAA CCC GCA GGC UGU UdT dT-3'and5'- UGA GAU CCU GGU CAU GAG GdT dT-3') were transfected into HUVECs by using Lipofectamine 2000 reagent (Invitrogen, Carlsbad, CA) in serum-free OPTI-MEM according to the manufacturer's instructions. Knockdown efficiency was evaluated $48 \mathrm{~h}$ after transfection by measuring protein levels in cell lysates using immunoblotting assays.

\section{Western blotting assay}

Cells were washed twice with PBS on the ice, and then lysed with ice-cold RIPA lysis buffer containing 1\% Phenylmethanesulfonyl fluoride (PMSF) and complete protease inhibitor cocktail (Beyotime Institute of Biotechnology, China). The lysates were then clarified by centrifugation at $12,000 \mathrm{~g}$ for $10 \mathrm{~min}$ at $4{ }^{\circ} \mathrm{C}$. The protein extracts were separated by SDS-PAGE and transferred to PVDF membranes. Membranes were blocked in $5 \%$ non-fat milk at room temperature for $1 \mathrm{~h}$, and then incubated with the diluted primary antibody in TBST buffer at $4^{\circ} \mathrm{C}$ for overnight. After incubation, membranes were washed three times for $10 \mathrm{~min}$ at room temperature with TBST buffer and incubated with the appropriate peroxide-conjugated secondary antibody for $1 \mathrm{~h}$. Membranes were then rinsed three times for 10 min with TBST buffer. Target proteins were detected using ECL reagent (Thermo Scientific, Rockford, IL) according to the manufacturer's instructions.

\section{Pulldown assays}

To perform the pulldown assay with nucleotide-free Rac1 mutant (G15ARac1), HUVECs were lysed in a buffer consisting of 1\% Triton X-100, 20 mM HEPES, pH 7.4, $150 \mathrm{mM} \mathrm{NaCl}, 5 \mathrm{mM} \mathrm{MgCl}$, and protease inhibitors. Lysates were cleared by centrifugation at 12,000 $\times \mathrm{g}$ for $10 \mathrm{~min}$. Equal amounts of protein were incubated at $4{ }^{\circ} \mathrm{C}$ for 60 min with GST or GST fusion proteins containing nucleotide-free Rac1 protein (G15ARac1) bound to glutathione- Sepharose. After $1.5 \mathrm{~h}$ of incubation, the beads were washed 3 times with lysis buffer. Samples were immunoblotted using antibodies against Vav2, Sos1, $\beta$-PIX and Dock180.

For detection of active Rac1, equal volumes of total cellular protein were incubated with GST-PBD beads captured on MagneGST glutathione particles (Promega, Madison, WI) at $4{ }^{\circ} \mathrm{C}$ with constant rotation for $60 \mathrm{~min}$. The beads were washed three times with washing buffer $(4.2 \mathrm{mmol} / \mathrm{L} \mathrm{Na2HPO4,} 2 \mathrm{mmol} / \mathrm{L}$ $\mathrm{KH} 2 \mathrm{PO} 4,280 \mathrm{mmol} / \mathrm{L} \mathrm{NaCl}$, and $10 \mathrm{mmol} / \mathrm{L} \mathrm{KCl}, \mathrm{pH}$ 7.2). At the end of this period, beads were captured by the magnet in a magnetic stand. After washing three times with ice-cold buffer, beads were resuspended in Laemmli buffer, boiled, and subjected to immunoblotting analysis. SDS-PAGE and immunoblotting were performed by using standard methods. 


\section{Cellular Physiology Cell Physiol Biochem 2017;41:1346-1359 \\ \begin{tabular}{l|l} 
and Biochemistry Published onIIne:IVarch 09, 2017 & $\begin{array}{l}\text { DOI: } 2017 \text { The Author(s). Published by S. Karger AG, Basel } \\
\text { www.karger.com/cpb }\end{array}$
\end{tabular}}

Ju et al:: Autocrine VEGF and IL-8 Promote Migration of Endothelial Cells

Enzyme-linked immunosorbent assay (ELISA)

At predetermined experimental time points $(0,12,24,48,72 \mathrm{~h})$ supernatant from HUVECs were collected and centrifuged at $1,000 \mathrm{~g}$ for $10 \mathrm{~min}$ and stored at $-80^{\circ} \mathrm{C}$. VEGF and IL-8 were measured by ELISA according to the kit's protocol (R\&D, MN, USA).

Statistical analysis

The data were analyzed using Student's t test using the SPSS statistical software package version 13 (SPSS Inc., Chicago, IL). All data were expressed as mean \pm SD. For all analyses a two-sided p value of less than 0.05 was deemed statistically significant.

\section{Results}

Autocrine VEGF and IL-8 increase the migration ability of HUVECS

To evaluate the effect of conditioned medium (CM) on migration ability of HUVECs, cells were plated on the upper cell culture inserts, with CM or plain medium (PM) in the lower chambers in the transwell assay. The results showed that the number of cells that were able to migrate the transwell membrane and came to its bottom side was $\sim 3.7$ fold higher in $\mathrm{CM}$ than in PM (Fig. 1 A), suggesting the migration potentiating effect of CM on HUVECs. In order to find out the putative mediators of CM-induced migration in HUVECs, we first examined the automatic secretion of VEGF and IL-8 protein by HUVECs. The result showed that HUVECs secreted VEGF and IL-8 protein a time-dependent manner, with the highest level at $48 \mathrm{~h}$ (Fig. $1 \mathrm{~B}$ ). To investigate the role of autocrine VEGF and IL-8 in HUVECs migration, we treated CM with neutralising antibodies against VEGF and IL-8 followed by transwell assay. Our results showed inhibition of motility in HUVECs when cultured in CM treated with neutralizing antibodies against VEGF and IL-8 (Fig. $1 \mathrm{C}$ ). Moreover, the magnitude of this inhibition was higher in CM treated with neutralising antibodies against VEGF and IL-8 in combination than neutralizing antibodies against VEGF or IL-8 alone (Fig. 1 C), suggesting VEGF and IL-8 as major mediators of CM-induced motility in HUVECs. To further confirm our finding, we then added recombinant human VEGF (rhVEGF) and recombinant human IL-8 (rhIL-8) in PM to check whether this can increase the motility of HUVECs comparable to that in CM. Our results revealed that, addition of VEGF and IL-8 in PM increased the motility compared to PM, however, this increase was found to be further higher when they were added in combination, implying the cooperativeness between these two cytokines (Fig. 1 D).

\section{Autocrine VEGF and IL-8-induced migration requires Rac1 activation}

Rac1, as an important small GTPase, has a pivotal role in mediating cell motility in a variety of cells including endothelial cells $[19,22]$, we wondered whether Rac1 activation was involved in the autocrine VEGF and IL-8-induced migration of HUVECs. We hence first investigated the effect of autocrine VEGF and IL-8 on Rac1 activation using Rac1 GTPase pull-down assay. We found that Rac1 activation was significantly induced 5 minutes after CM stimulation, peaked at 15min (Fig. 2A). Compared to CM control, in HUVECs grown in CM-treated with neutralising antibodies against VEGF and IL-8, the Rac1 activation levels were significantly lower (Fig. 2B). Furthermore, in CM treated with neutralising antibodies against VEGF and IL-8 together, the Rac1 activation level was further lower (Fig. 2B). These results suggest that autocrine VEGF and IL-8 induce Rac1 activation.

Next, we examined the effect of Rac1 activation on autocrine VEGF and IL-8-induced cell migration. Depletion of Rac1 by siRNA resulted in an approximately $70 \%$ reduction in the protein level of Rac1 (Fig. 2C), which was accompanied by a remarkable inhibition of autocrine VEGF and IL-8-induced cell migration (Fig. 2D). Taken together, these experiments demonstrate that Rac1 activation is required for autocrine VEGF and IL-8-induced migration of HUVECs.

Vav2 is required for autocrine VEGF and IL-8-induced Rac1 activation and cell migration

It has been shown that activation of Rac1 is regulated by guanine nucleotide exchange factors (GEFs) [26]. To determine which exchange factors activate Rac1 downstream of 
Fig. 1. Effect of autocrine VEGF and IL- 8 on the migration of HUVECs. (A) Comparison of the motility of HUVECs in culture medium of CM and PM. Data were presented as mean \pm SD. ${ }^{* *} \mathrm{p}<0.05$ in cultures with $\mathrm{CM}$ relative to cultures with PM. (B) VEGF and IL-8 secreted by HUVECs in the complete media were measured by ELISA for the indicated times. (C) Effect of autocrine VEGF and IL-8 on the migration of HUVECs. HUVECs were seeded in the upper compartment of the migration chamber and CM pre-treated with neutralising antibodies against VEGF and IL-8 for $1 \mathrm{~h}$ in the lower chamber. Migration assay was performed after $18 \mathrm{~h}$ and expressed as fold increase (relative to CM control) by calculating cell number. Data were presented as mean $\pm \mathrm{SD}$, $* * \mathrm{p}<0.05$ in cultures with CM plus antibodies against VEGF or/and IL-8 relative to cultures with $\mathrm{CM}$ alone. (D) HUVECs were seeded in the upper compartment of the migration chamber and PM, PM added with rhVEGF or/and rhIL8 in the lower chamber. Migration assay was performed after $18 \mathrm{~h}$ and expressed as fold increase (relative to PM control) by calculating cell number. Data were presented as mean $\pm \mathrm{SD},{ }^{* *} \mathrm{p}<0.05$ in cultures with PM plus rhVEGF or/and rhIL-8 relative to cultures with PM alone.

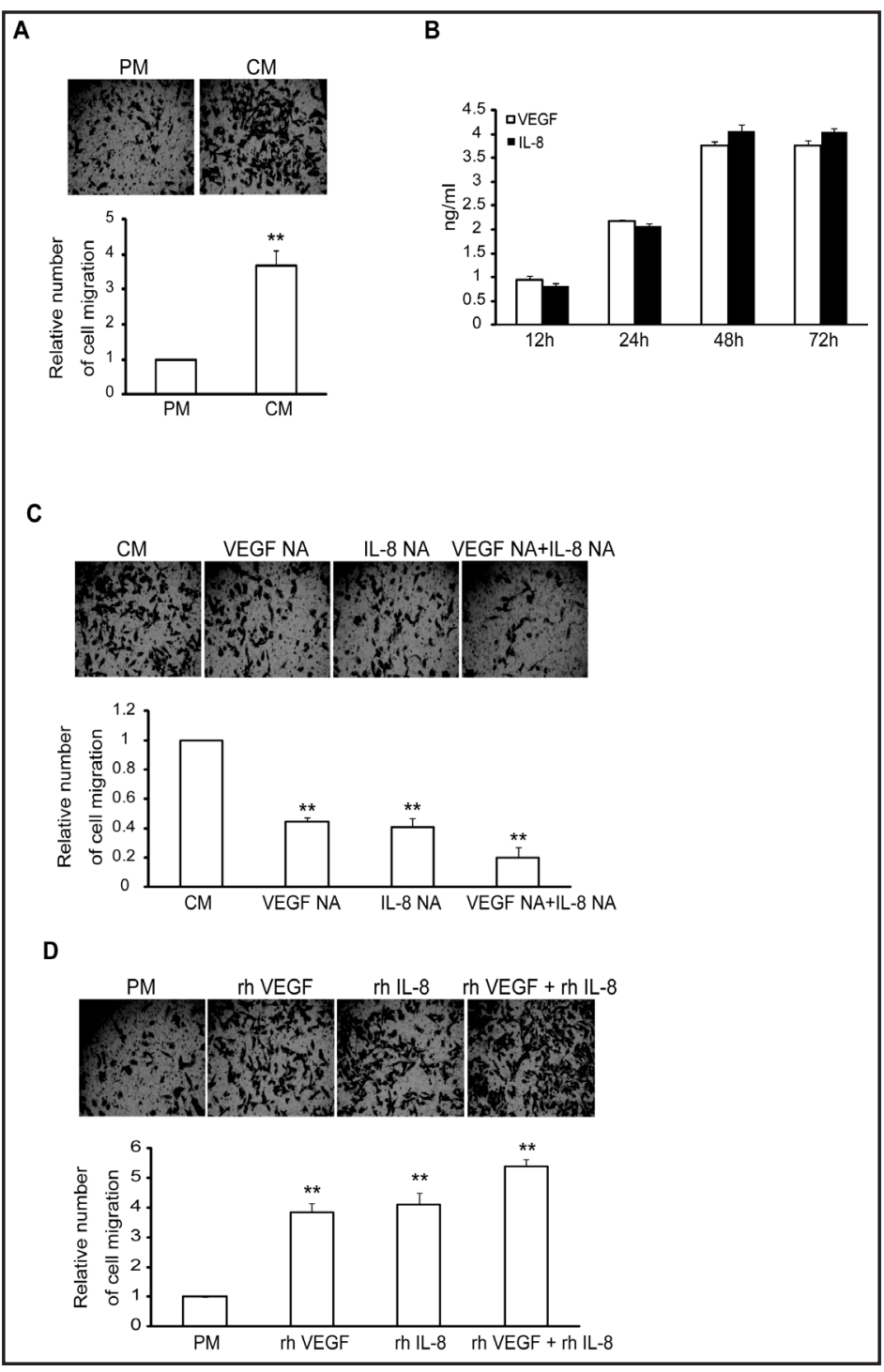

D

autocrine VEGF and IL-8, we first investigated the endogenous expression of candidate Rac1 GEFs (Vav2, Tiam1, $\beta$-PIX, Sos-1 and D0CK180) in HUVECs. We found that Vav2, $\beta$-PIX, Sos1 and DOCK180 were readily detectable in HUVECs, whereas Tiam1 was not detectable, indicating an extremely low expression level in HUVECs (Fig. 3A). To determine which GEF might be involved in Rac1 activation downstream from autocrine VEGF and IL-8, we used nucleotide-free Rac1 in a pulldown strategy. Using a GST fusion protein of the G15ARac1 mutant, we found that CM treatment increased the association of Vav2 with G15ARac1 (Fig.3B). G15ARac1 failed to pull down Sos1 after CM treatment. Low levels of $\beta$-PIX and Dock180 were detected binding to the G15ARac1 mutant, but the level of binding was not increased by CM treatment (Fig. 3B). Furthermore, when we inhibited autocrine of VEGF and IL-8 with antibodies against VEGF and IL-8 in CM, CM treatment did not stimulate the interaction between Vav2 and G15ARac1 (Fig. 3C). These results indicate that of the GEFs examined only Vav2 increases its association with G15ARac1 after autocrine IL-8 and VEGF stimulation.

To further study the role of Vav2 in autocrine VEGF and IL-8-induced Rac1 activation and cell migration, we used siRNA against Vav2 to decrease Vav2 expression in HUVECs. Silencing Vav2 resulted in a decrease of Vav2 protein expression (Fig. 3D), and largely 




\section{B}

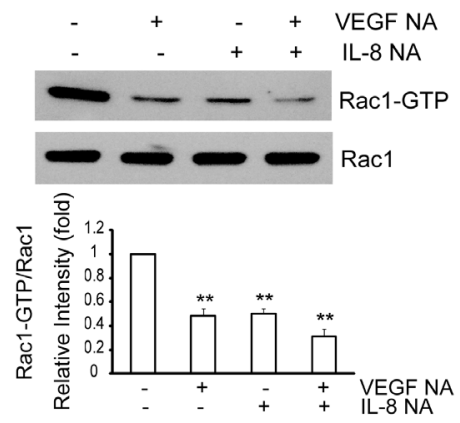

C

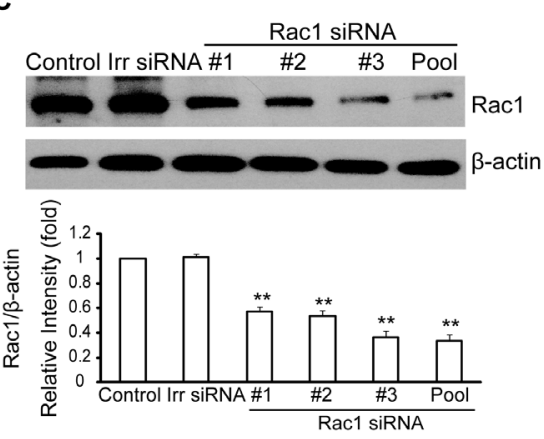

D

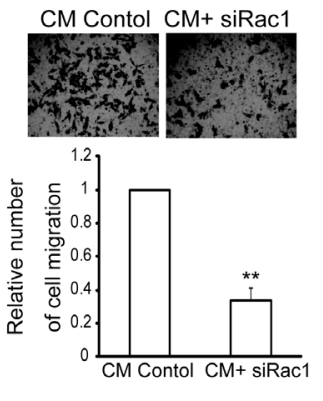

Fig. 2. Effect of Rac1 activation on autocrine VEGF and IL-8-induced cell migration. (A) Effect of CM stimulation on activity of Rac1. Serum-starved HUVECs were treated with CM for the indicated time periods. After stimulation, cells were analyzed for Rac1 activation by pulldown assays. Data were presented as mean \pm SD. ${ }^{* *}$ p $<0.05$ in cultures with CM relative to cultures without CM. (B) Effect of autocrine VEGF and IL-8 on Rac1 activation. HUVECs treated with CM and CM neutralized for VEGF and IL-8 harvested at 15 min and lysates were prepared and processed for pulldown for Rac1-GTP. Data were presented as mean \pm SD. ** $\mathrm{p}<0.05$ in cultures with CM plus antibodies against VEGF or/and IL-8 relative to cultures with CM alone. (C) Effect of Rac1 siRNA on the expression of Rac1. HUVECs were transiently transfected with appropriate Rac1 siRNA, Rac1 siRNA pool, scrambled siren (Irr siRNA) or not for $48 \mathrm{~h}$. Cells were analyzed by immunoblotted with Rac1 antibody. Data were presented as mean \pm SD. ${ }^{* *} \mathrm{p}<0.05$ in cultures transiently transfected with Rac1 siRNA relative to non-transfected cultures. (D) Effect of Rac1 siRNA on CM-stimulated cell migration. HUVECs transfected with Rac1 siRNA as indicated were subjected to a transwell assay in the presence of CM for 18 h. ${ }^{* *} \mathrm{p}<0.05$ in cultures transiently transfected with Rac1 siRNA and treated with CM relative to non-transfected cultures and treated with CM.

inhibited autocrine VEGF and IL-8-induced Rac1 activation (Fig. 3E) and cell migration (Fig. $3 \mathrm{~F}$ ). Taken together, these experiments demonstrate that Vav2 expression is required for autocrine VEGF and IL-8-induced Rac1 activation and cell migration in HUVECs.

Src is the upstream regulator of Vav2-mediated Rac1 activation in autocrine VEGF and IL-8-induced cell migration

The non-receptor tyrosine kinase Src is widely recognized as a key mediator in Rac1 activation and is important for endothelial cell migration [36, 37].We hypothesized that active Src might also be involved in autocrine VEGF and IL-8-induced Rac1 activation and cell migration in HUVECs. Therefore, we determined whether Src activation was required for autocrine VEGF and IL-8-induced Rac1 activation and cell migration. Immunoblotting showed a visible sign of basal active Src and a maximal effect at 5 min after CM treatment 
Fig. 3. Vav2 is required for autocrine VEGF and IL-8-induced Rac1 activation and cell migration. (A) Endogenous expression of candidate Rac1 GEFs in HUVECs. Equivalent levels of protein from HUVECs or COS7 cells were probed with antibodies against other Rac1 GEFs, Vav2, $\beta$-PIX, Tiam1, Sos1 and Dock180. $\beta$-actin indicated equal amount of protein was loaded. (B) CM stimulates Vav2-Rac1 complex formation. HUVECs were serum-starved and then treated CM for 15 min, lysed, and lysates were incubated with the nucleotide-free Rac1 mutant fused to GST (GST-G15ARac1) on glutathione-Sepharose beads for $60 \mathrm{~min}$ at $4^{\circ} \mathrm{C}$. Antibodies against Vav2, $\beta$-PIX, Sos1 and Dock180 were used to detect binding of these proteins to G15ARac1. (C) Effect of autocrine VEGF and IL-8 on Vav2-Rac1 complex formation. HUVECs treated with CM and CM neutralized for

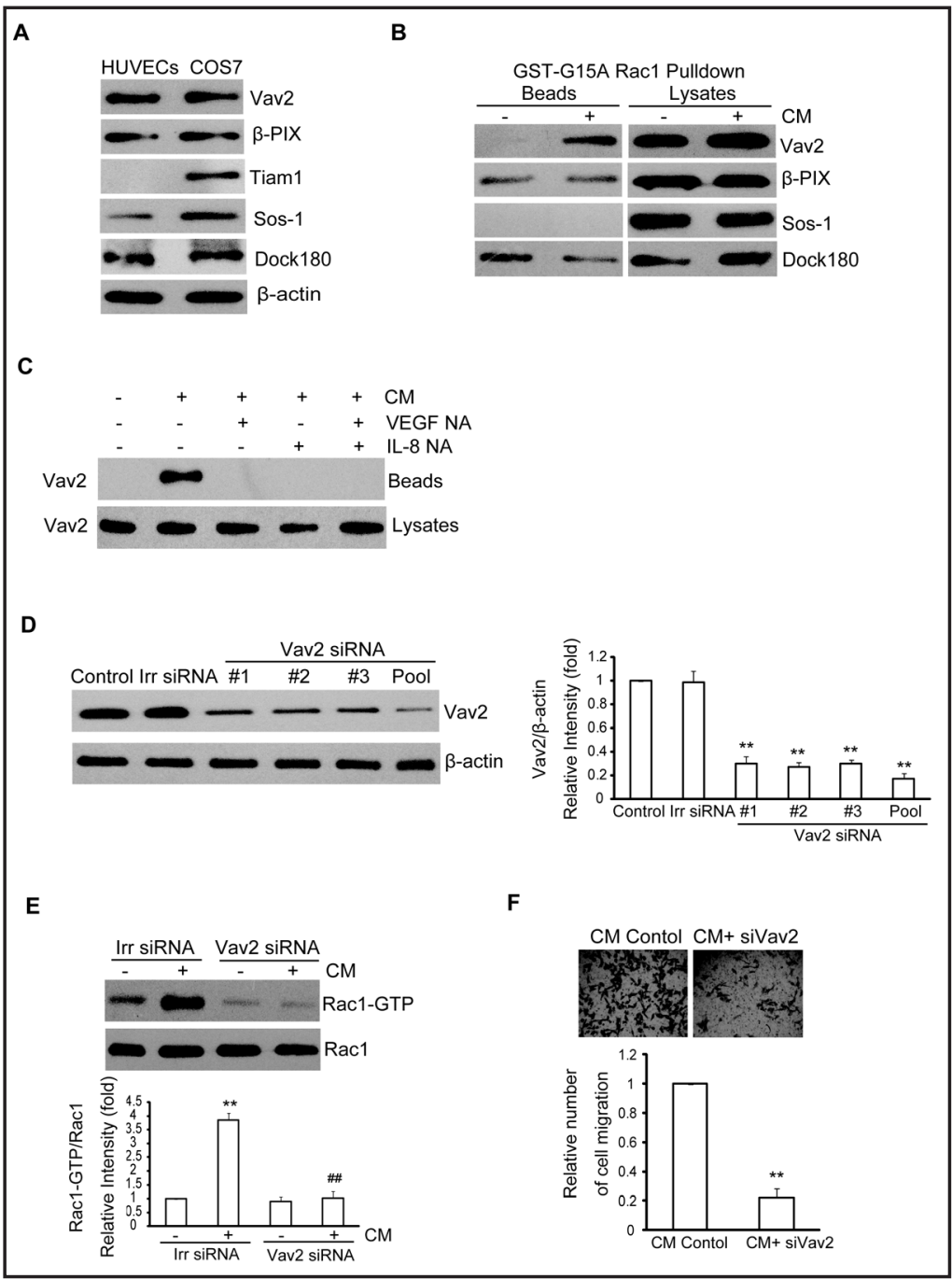

VEGF and IL-8 harvested at 15 min and lysates were prepared and processed for pulldown as described in (B). (D) Effect of Vav2 siRNA on the expression of Vav2. HUVECs were transiently transfected with appropriate Vav2 siRNA, Vav2 siRNA pool, scrambled siren (Irr siRNA) or not for $48 \mathrm{~h}$. Cells were analyzed by immunoblotted with Rac1 antibody. Data were presented as mean \pm SD. ${ }^{* *} \mathrm{p}<0.05$ in cultures transiently transfected with Vav2 siRNA relative to non-transfected cultures. (E) Effect of Vav2 siRNA on CM-stimulated activation of Rac1. HUVECs were transiently transfected with Vav2 siRNA or not, then stimulated with CM or not. After stimulation, cells were analyzed by pulldown with Rac1 antibody. Data were presented as mean $\pm \mathrm{SD}$. ${ }^{* *} \mathrm{p}<0.05$, referring to the difference between cells treated with and without $\mathrm{CM}$. $\# \# \mathrm{p}<0.05$, referring to the difference between the cells transfected with Vav2 siRNA and the cells transfected with scrambled siRNA (irr siRNA) plus CM. (F) Effect of Vav2 siRNA on CM-stimulated cell migration. HUVECs transfected with Vav2 siRNA as indicated were subjected to a transwell assay in the presence of CM for $18 \mathrm{~h}$. Data were presented as mean \pm SD. ${ }^{* *} p<0.05$ in cultures transiently transfected with Vav2 siRNA and treated with CM relative to non-transfected cultures and treated with $\mathrm{CM}$.

(Fig. 4A). Compared to CM control, in HUVECs grown in CM-treated with neutralising antibodies against IL-8 and VEGF, the Src activation levels were significantly lower (Fig. 4B). Importantly, the Src activation level was further lower in HUVECs grown in CM treated with neutralising antibodies against IL-8 and VEGF together (Fig. 4B). These results indicate that autocrine VEGF and IL-8 induce Src activation.

\section{KARGER}


Fig. 4. The activation of Src is required for autocrine VEGF and IL8-induced Rac1 activation and cell migration. (A) Effect of CM stimulation on phosphorylation of Src. Serum-starved HUVECs were treated with $\mathrm{CM}$ for the indicated time periods. After stimulation, cells were analyzed for Src activation by immunoblotted with p-Src (Tyr418) or total Src antibodies. Data were presented as mean \pm SD. ${ }^{* *} \mathrm{p}<0.05$ in cultures with CM relative to cultures without CM. (B) Effect of autocrine VEGF and IL-8 on Src activation. HUVECs treated with $\mathrm{CM}$ and CM neutralized for VEGF and IL-8 harvested at 5 min and lysates were prepared and processed for immunoblotting for $\mathrm{p}$-Src or total Src. Data were presented as mean \pm SD of. ${ }^{* *} \mathrm{p}<0.05$ in cultures with $\mathrm{CM}$ plus antibodies against VEGF or/ and IL-8 relative to cultures with CM alone. (C) Effect of PP2 on phosphorylation of Vav2 at Tyr172. Cells were incubated for $15 \mathrm{~min}$ in the absence or presence of $30 \mu \mathrm{M}$ PP2 prior to CM treatment. After stimulation, cells were analyzed by immunoblotting with p-Vav2 or total Vav2 antibodies. Data were presented as mean $\pm \mathrm{SD}$. ${ }^{* *} \mathrm{p}<0.05$, referring to the difference between cells treated with and without $\mathrm{CM}$. $\# \# \mathrm{p}<0.05$, referring to the difference between the cells treated with CM plus PP2 and the cells treated with CM alone. (D) Effect of PP2 on activation of Rac1. Cells were incubated for $15 \mathrm{~min}$ in the absence or presence of $30 \mu \mathrm{M}$ PP2 prior to CM treatment. After stimulation, cells were analyzed for Rac1 activation. Data were presented as mean \pm SD. ${ }^{* *} \mathrm{p}<0.05$, referring to the difference between cells treated with and without $\mathrm{CM}$. $\# \# \mathrm{p}<0.05$, referring to the difference between the cells treated with CM plus PP2 and the cells treated with CM alone. (E) Effect of PP2 on CM-stimulated cell migration. HUVECs pretreated with $30 \mu \mathrm{M}$ PP2 were subjected to a transwell assay in the presence of CM for $18 \mathrm{~h}$. Data were presented as mean $\pm \mathrm{SD}$. ${ }^{* *} \mathrm{p}<0.05$ in cells treated with CM plus PP2 relative to treated with CM alone.

To investigate the role of Src in autocrine VEGF and IL-8-induced Rac1 activation and cell migration, Src kinase activity was blocked with PP2, a Src inhibitor. As shown in Fig. 4C, CM treatment induced phosphorylation of Vav2 on tyrosine 172. This phosphorylation was blocked by PP2, suggesting that this phosphorylation depended on Src kinase activity. The Src inhibitor PP2 also abolished CM-induced Rac1 activation (Fig. 4D) as well as cell migration (Fig. 4E). Taken together, these data indicate that autocrine VEGF and IL-8induced tyrosine phosphorylation of Vav2 is regulated by Src and that Src kinase activity is required for activation of Rac1 and cell migration which induced by autocrine VEGF and IL-8 in HUVECs.

PAK1 as a downstream target of Rac1 in mediating autocrine VEGF and IL-8-induced migration

Previous reports have shown that P21-activated kinase 1 (PAK1), a main downstream effector of Rac1, is involved in regulating endothelial migration and angiogensis [33, 34]. Therefore, we examined whether PAK1 activation also occurred in our system. Western 
Fig. 5. PAK1 is a downstream target of Rac1 and its activation is required for autocrine VEGF and IL-8induced cell migration. (A) Effect of CM stimulation on phosphorylation of PAK1. Serum-starved HUVECs were treated with $\mathrm{CM}$ for the indicated time periods. After stimulation, cells were analyzed for PAK1 activation by immunoblotted with p-PAK1 (Thr423) or total PAK1 antibodies. Data were presented as mean \pm SD. ${ }^{* *} \mathrm{p}<0.05$ in cultures with CM relative to cultures without CM. (B) Effect of autocrine VEGF and IL-8 on PAK1 activation. HUVECs treated with $\mathrm{CM}$ and $\mathrm{CM}$ neutralized for VEGF and IL-8 harvested at $30 \mathrm{~min}$ and lysates were prepared and processed for immunoblotting for p-PAK1 or total PAK1. Data were presented as mean \pm SD. ${ }^{* *} \mathrm{p}<0.05$ in cultures with CM plus anti-

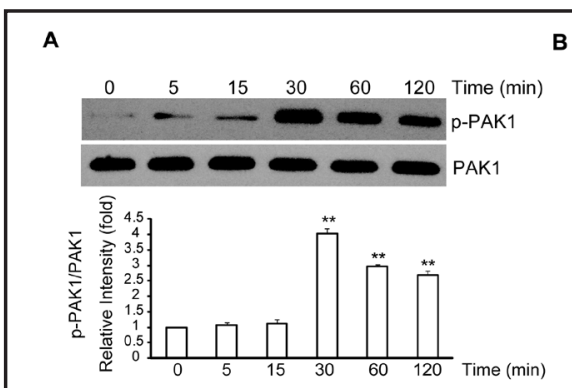

B

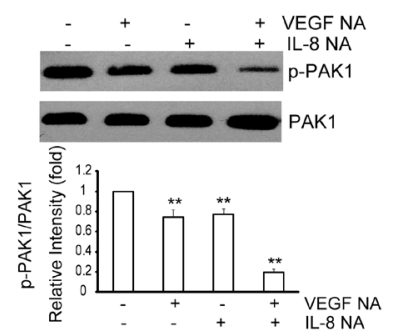

C

D
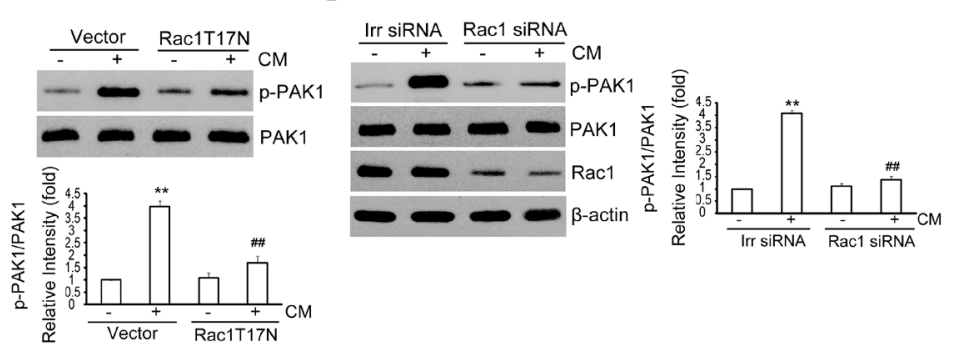

E

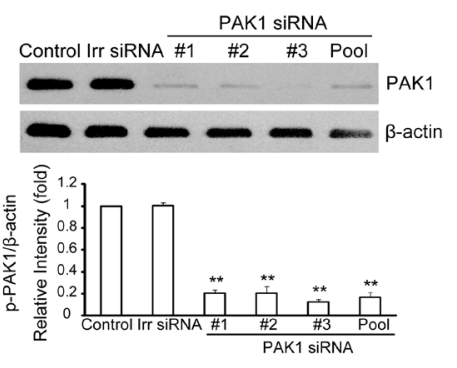

F
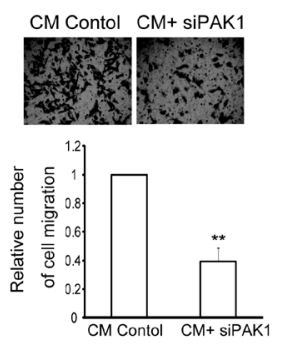

bodies against VEGF and IL-8 relative to cultures with CM alone. (C) Effect of Rac1T17N on CM-stimulated activation of PAK1. HUVECs were transiently transfected with Rac1T17N or empty vector, then stimulated with CM or not. After stimulation, cells were analyzed by immunoblotting with p-PAK1 and total PAK1 antibodies. Data were presented as mean $\pm \mathrm{SD}$.** $\mathrm{p}<0.05$, referring to the difference between cells treated with and without $\mathrm{CM}$. \#\#p<0.05, referring to the difference between the cells transfected with Rac1T17N plus CM and the cells transfected with empty vector plus CM. (D) Effect of Rac1 siRNA on CM-stimulated activation of PAK1. HUVECs were transiently transfected with Rac1 siRNA or scrambled siRNA (irr siRNA), then stimulated with CM or not. After stimulation, cells were analyzed by immunoblotting. Data were presented as mean $\pm \mathrm{SD}$. ${ }^{* *} \mathrm{p}<0.05$, referring to the difference between cells treated with and without $\mathrm{CM}$. \#\# $<<0.05$, referring to the difference between the cells transfected with Rac1 siRNA plus CM and the cells transfected with Irr siRNA plus CM. (E) Effect of PAK1 siRNA on the expression of PAK1. HUVECs were transiently transfected with appro- priate PAK1 siRNA, PAK1 siRNA pool, scrambled siren (Irr siRNA) or not for $48 \mathrm{~h}$. Cells were analyzed by immunoblotted with PAK1 antibody. Data were presented as mean \pm SD. ${ }^{* *} \mathrm{p}<0.05$ in cultures transiently transfected with PAK1 siRNA relative to non-transfected cultures. (F) Effect of PAK1 siRNA on CM-stimulated cell migration. HUVECs transfected with PAK1 siRNA as indicated were subjected to a transwell assay in the presence of CM for $18 \mathrm{~h}$. Data were presented as mean $\pm \mathrm{SD}$. ${ }^{* *} \mathrm{p}<0.05$ in cultures transiently transfected with PAK1 siRNA and treated with CM relative to non-transfected cultures and treated with CM.

blotting analysis showed that the amount of phosphorylated PAK1 at Thr 423 was increased significantly after CM stimulation with maximal activation at $30 \mathrm{~min}$. (Fig. 5A). HUVECs grown in CM-treated with neutralising antibodies against VEGF and IL-8 showed a decreased PAK1 activation in comparison with control cells (Fig. 5B). The PAK1 activation level was further lower in HUVECs grown in CM treated with neutralising antibodies against VEGF and IL-8 together (Fig. 5B). 
To determine whether autocrine VEGF and IL-8-stimulated PAK1 activity was Rac1 activation-dependent, we blocked Rac1 activity by transfecting HUVECs with Rac1 T17N (an inactive mutant of Rac1) and examined PAK1 activation. We found that, while CM treatment significantly increased PAK1 activity in cells expressing the empty vector, it failed to do so in cells expressing Rac1 T17N (Fig. 5C). We also examined the effect of Rac1 on autocrine VEGF and IL-8-induced PAK1 activation by knocking down Rac1 expression with appropriate siRNAs in HUVECs. We found that, compared with scrambled siRNA, siRNA against Rac1 noticeably attenuated CM-induced PAK1 activation (Fig. 5D).

To further determine whether autocrine VEGF and IL-8 stimulate cell migration in a PAK1-dependent manner, we investigated migration of HUVECs after transfecting these cells with the PAK1 siRNA, which reduced the protein level of PAK1 by approximately $80 \%$, as assessed by immunoblotting (Fig. 5E) and significantly reduced CM-induced migration of HUVECs (Fig. 5F). These experiments demonstrate that PAK1 as a downstream target of Rac1 in mediating autocrine VEGF and IL-8-induced migration.

\section{Discussion}

Emerging findings indicate that VEGF and IL-8 signaling are implicated in regulating endothelial cell migration $[22,38,39]$. It has been reported that VEGF and IL-8 can promote endothelial cell migration in an autocrine manner $[15,16]$. In our system, cell migration rate of HUVECs was accelerated after CM treatment compared with that of the control. At the same time, the effect of CM was inhibited by neutralising antibodies against VEGF and IL-8. Therefore, autocrine VEGF and IL-8 directly activate the migration of HUVECs, which is a critical step for angiogenesis. Based on this, the signaling mechanisms underlying the effect of autocrine VEGF and IL-8 on promoting endothelial cell migration were investigated.

The activation of Rac1, a small G-protein, mediates migration of various types of cell, including endothelial cells [40]. A primary observation in the present study is that CM induced activation of Rac1 in a time-dependent fashion in HUVECs. Moreover, we observed that CM-induced Rac1 activation was suppressed by neutralising antibodies against VEGF and IL-8, indicating that autocrine VEGF and IL-8 can activate Rac1. It has been reported that IL-8 promotes endothelial cell migration by increasing the activity of Rac1 [22]. Caron et al. have shown that activation of Rac1 mediates VEGF-stimulated endothelial cell migration [41]. Our results found that when Rac1 activation was blocked, autocrine VEGF and IL-8stimulated cell migration was dramatically diminished. Therefore, our results suggest that Rac1 activation serves as a mediator of autocrine VEGF and IL-8-stimulate endothelial cell migration.

We next examined the potential activators for Rac1 in our system. Like all GTPases, the Rac1 is under tight spatial control, which is mediated by guanine nucleotide exchange factors (GEFs) and GTPase-activating proteins (GAPs) that catalyse GTP exchange and hydrolysis, respectively [23]. Specific GEF allows Rac1 to be activated in specific signal transduction pathways and coordinate more elaborate responses to specific demands at localized cellular sites [26, 42]. In this study, we identified the exchange factor Vav2 as mediating autocrine VEGF and IL-8 signaling to Rac1. Using a nucleotide-free G15ARac1 mutant, we showed that Vav2 interacts with Rac1 after CM stimulation and this association was inhibited by neutralising antibodies against VEGF and IL-8. Moreover, knockdown of Vav2 not only inhibited the increase in Rac1 activity in response to CM stimulation, but also inhibited CM stimulated migration. Together these results indicate that Vav2 plays a critical role in the response to autocrine VEGF and IL-8. A previous study showed that Vav2 activated Rac1 after VEGF stimulation through the VEGFR-2 signaling in endothelial cells [43]. On the other hand, Work from other labs has indicated that IL-8 promotes the activation of VEGF receptors in an autocrine fashion by controlling the expression of VEGF in endothelial cells [44].Therefore, it is possible that Vav2 mediates autocrine VEGF and IL-8-induced Rac1 activation through VEGFR-2 signaling in our system. Further studies are needed to 
decipher whether expression and activation of VEGFR-2 is required for Vav2-mediated Rac1 activation in response to autocrine VEGF and IL-8 in endothelial cells.

The Src tyrosine kinase has been identified as a key mediator in endothelial cell migration [45]. Signals initiated by VEGF or IL-8 are transduced into cells through the activation of Src $[29,30]$. A previous study shows that $\mathrm{Src}$ is an upstream of Vav2 in controlling Rac1 activation [43].The results of our present study show that CM-induced cell migration was associated with an increase in Src activity. CM-induced Src activation was suppressed by neutralising antibodies against VEGF and IL-8, indicating that autocrine VEGF and IL-8 can activate Src. Moreover, our results showed that Src inhibitor PP2 could inhibit not only autocrine VEGF and IL-8-induced phosphorylation of Vav2, but also Rac1 activation as well as migration of HUVECs. Consequently, we conclude that Src is an upstream component of Vav2/Rac1 signaling pathway in autocrine VEGF and IL-8-stimulated endothelial cell migration. Previous studies have shown that Src is critical for Rac1 activity downstream of growth factor receptors in diverse cell types [46]. Src has also been shown to be implicated in cell migration mediated by adhesion molecule and Rac1-dependent endothelial cell migration $[37,47]$. Meanwhile, our data indicate that Src is capable of increasing Rac1 activity through the Vav2 signaling, which is associated the autocrine VEGF and IL-8 with endothelial cell migration. Therefore, we conclude that Src is commonly used by cells to activate Rac1 and promote migration in response to diverse signaling pathways.

We then focused on the potential downstream effector of Rac1 that is associated with autocrine VEGF and IL-8-trigged migration of HUVECs. PAK1 is widely recognized as a potential modulator of endothelial cell migration, permeability, and angiogensis [33, 34]. Previous studies have shown that PAK1 is a downstream mediator of Rac1 in controlling tumor migration and invasion [48]. Furthermore, the expression of a constitutively active form of PAK1 in endothelial cells has been shown to induce the rapid formation of lamellipodia, filopodia, and dorsal ruffles, as well as an increase in the reorganization of actin cytoskeleton and cell migration [33]. This prompted us to investigated whether Rac1 mediates autocrine VEGF and IL-8-induced endothelial cell migration through PAK1 activation. Our results showed that CM triggers a rapid stimulation of PAK1 activity. CMinduced PAK1 activation was suppressed by neutralising antibodies against VEGF and IL8, indicating that autocrine VEGF and IL-8 can activate PAK1. Preventing Rac1 activity by Rac1 T17N and Rac1 siRNA decreased the PAK1 activation-induced by autocrine VEGF and IL-8, suggesting that autocrine VEGF and IL-8-induced Rac1 activity was responsible for activation of PAK1 in endothelial cells. When PAK1 activity was blocked by PAK1 siRNA, autocrine VEGF and IL-8-stimulated cell migration was dramatically diminished. Therefore, our results suggest that PAK1 activation serves as a downstream mediator of Rac1 in regulating autocrine VEGF and IL-8-stimulated endothelial cell migration.

In summary, we present the first direct evidence here that autocrine VEGF and IL-8 promote cell migration of HUVECs via Src/Vav2/Rac1/PAK1 signaling. This finding reveals the molecular mechanism in the increase of endothelial cell migration induced by autocrine VEGF and IL-8, which may lead to the development of new therapies that can be applied in pathological conditions involving increase in endothelial cell migration.

\section{Acknowledgments}

This work was supported by the Project of Nanjing medical science and technology development foundation (ZKX13038).

\section{Disclosure Statement}

The authors have nothing to disclose. 


\section{Cellular Physiology Cell Physiol Biochem 2017;41:1346-1359

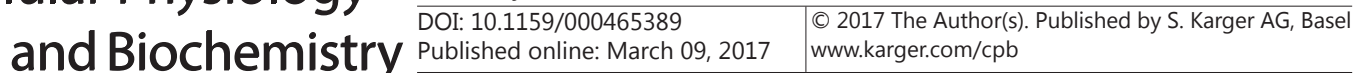

Ju et al:: Autocrine VEGF and IL-8 Promote Migration of Endothelial Cells

\section{Reference}

1 Huang S, He P, Peng X, Li J, Xu D, Tang Y: Pristimerin inhibits prostate cancer bone metastasis by targeting pc-3 stem cell characteristics and vegf-induced vasculogenesis of bm-epcs. Cell Physiol Biochem 2015;37:253-268.

2 Betz C, Lenard A, Belting HG, Affolter M: Cell behaviors and dynamics during angiogenesis. Development 2016;143:2249-2260.

3 Bruns H, Kazanavicius D, Schultze D, Saeedi MA, Yamanaka K, Strupas K, Schemmer P: Glycine inhibits angiogenesis in colorectal cancer: Role of endothelial cells. Amino Acids 2016;48:2549-2558.

-4 Hang TC, Tedford NC, Reddy RJ, Rimchala T, Wells A, White FM, Kamm RD, Lauffenburger DA: Vascular endothelial growth factor (vegf) and platelet (pf-4) factor 4 inputs modulate human microvascular endothelial signaling in a three-dimensional matrix migration context. Mol Cell Proteomics 2013;12:37043718.

-5 Cai H, Xue Y, Li Z, Hu Y, Wang Z, Liu W, Li Z, Liu Y: Roundabout4 suppresses glioma-induced endothelial cell proliferation, migration and tube formation in vitro by inhibiting vegr2-mediated pi3k/akt and fak signaling pathways. Cell Physiol Biochem 2015;35:1689-1705.

-6 Abhinand CS, Raju R, Soumya SJ, Arya PS, Sudhakaran PR: Vegf-a/vegfr2 signaling network in endothelial cells relevant to angiogenesis. J Cell Commun Signal 2016;10:347-354.

-7 Chamorro-Jorganes A, Lee MY, Araldi E, Landskroner-Eiger S, Fernandez-Fuertes M, Sahraei M, Quiles Del Rey M, van Solingen C, Yu J, Fernandez-Hernando C, Sessa WC, Suarez Y: Vegf-induced expression of mir17-92 cluster in endothelial cells is mediated by erk/elk1 activation and regulates angiogenesis. Circ Res 2016;118:38-47.

8 Li A, Dubey S, Varney ML, Dave BJ, Singh RK: Il-8 directly enhanced endothelial cell survival, proliferation, and matrix metalloproteinases production and regulated angiogenesis. J Immunol 2003;170:3369-3376.

-9 Wang J, Wang Y, Wang S, Cai J, Shi J, Sui X, Cao Y, Huang W, Chen X, Cai Z, Li H, Bardeesi AS, Zhang B, Liu M, Song W, Wang M, Xiang AP: Bone marrow-derived mesenchymal stem cell-secreted il-8 promotes the angiogenesis and growth of colorectal cancer. Oncotarget 2015;6:42825-42837.

10 Xie Q, Sun Z, Chen M, Zhong Q Yang T, Yi J: Il-8 up-regulates proliferative angiogenesis in ischemic myocardium in rabbits through phosphorylation of akt/gsk-3beta(ser9) dependent pathways. Int J Clin Exp Med 2015;8:12498-12508.

11 Jiang S, Li Y, Lin T, Yuan L, Li Y, Wu S, Xia L, Shen H, Lu J: Il-35 inhibits angiogenesis through vegf/ang2/tie2 pathway in rheumatoid arthritis. Cell Physiol Biochem 2016;40:1105-1116.

$\checkmark 12$ Vegran F, Boidot R, Michiels C, Sonveaux P, Feron O: Lactate influx through the endothelial cell monocarboxylate transporter mct1 supports an nf-kappab/il-8 pathway that drives tumor angiogenesis. Cancer Res 2011;71:2550-2560.

13 Chen RJ, Chen SU, Chou CH, Lin MC: Lysophosphatidic acid receptor 2/3-mediated il-8-dependent angiogenesis in cervical cancer cells. Int J Cancer 2012;131:789-802.

14 Chatterjee S, Heukamp LC, Siobal M, Schottle J, Wieczorek C, Peifer M, Frasca D, Koker M, Konig K, Meder L, Rauh D, Buettner R, Wolf J, Brekken RA, Neumaier B, Christofori G, Thomas RK, Ullrich RT: Tumor vegf:Vegfr2 autocrine feed-forward loop triggers angiogenesis in lung cancer. J Clin Invest 2013;123:17321740.

15 Jain HV, Jackson TL: A hybrid model of the role of vegf binding in endothelial cell migration and capillary formation. Front Oncol 2013;3:102.

16 Li X, Liu Y, Wang L, Li Z, Ma X: Unfractionated heparin attenuates lps-induced il-8 secretion via pi3k/akt/ nf-kappab signaling pathway in human endothelial cells. Immunobiology 2015;220:399-405.

17 Hall A: Rho family gtpases. Biochem Soc Trans 2012;40:1378-1382.

18 Herve JC, Bourmeyster N: Rho gtpases at the crossroad of signaling networks in mammals. Small GTPases 2015;6:43-48.

19 Mulherkar S, Uddin MD, Couvillon AD, Sillitoe RV, Tolias KF: The small gtpases rhoa and rac1 regulate cerebellar development by controlling cell morphogenesis, migration and foliation. Dev Biol 2014;394:3953.

20 Jeganathan N, Predescu D, Zhang J, Sha F, Bardita C, Patel M, Wood S, Borgia JA, Balk RA, Predescu S: Rac1mediated cytoskeleton rearrangements induced by intersectin-1s deficiency promotes lung cancer cell proliferation, migration and metastasis. Mol Cancer 2016;15:59. 


\section{Cellular Physiology Cell Physiol Biochem 2017;41:1346-1359

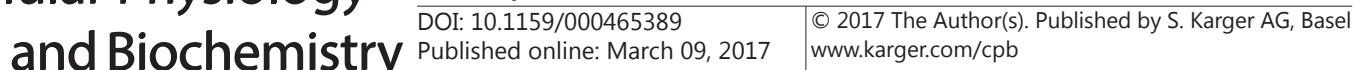

-21 Yang Y, Chen QH, Liu AR, Xu XP, Han JB, Qiu HB: Synergism of msc-secreted hgf and vegf in stabilising endothelial barrier function upon lipopolysaccharide stimulation via the rac1 pathway. Stem Cell Res Ther $2015 ; 6: 250$.

22 Lai Y, Shen Y, Liu XH, Zhang Y, Zeng Y, Liu YF: Interleukin-8 induces the endothelial cell migration through the activation of phosphoinositide 3-kinase-rac1/rhoa pathway. Int J Biol Sci 2011;7:782-791.

23 Cherfils J, Zeghouf M: Regulation of small gtpases by gefs, gaps, and gdis. Physiol Rev 2013;93:269-309.

24 Marei H, Malliri A: Gefs: Dual regulation of rac1 signaling. Small GTPases 2016:1-10.

25 Tolias KF, Bikoff JB, Burette A, Paradis S, Harrar D, Tavazoie S, Weinberg RJ, Greenberg ME: The rac1-gef tiam1 couples the nmda receptor to the activity-dependent development of dendritic arbors and spines. Neuron 2005;45:525-538.

-26 Marei H, Carpy A, Woroniuk A, Vennin C, White G, Timpson P, Macek B, Malliri A: Differential rac1 signalling by guanine nucleotide exchange factors implicates flii in regulating rac1-driven cell migration. Nat Commun 2016;7:10664.

27 Je DW, O YM, Ji YG, Cho Y, Lee DH: The inhibition of src family kinase suppresses pancreatic cancer cell proliferation, migration, and invasion. Pancreas 2014;43:768-776.

28 Nam K, Oh S, Lee KM, Yoo SA, Shin I: Cd44 regulates cell proliferation, migration, and invasion via modulation of c-src transcription in human breast cancer cells. Cell Signal 2015;27:1882-1894.

29 Liu CJ, Kuo FC, Wang CL, Kuo CH, Wang SS, Chen CY, Huang YB, Cheng KH, Yokoyama KK, Chen CL, Lu CY, Wu DC: Suppression of il-8-src signalling axis by 17beta-estradiol inhibits human mesenchymal stem cellsmediated gastric cancer invasion. J Cell Mol Med 2016;20:962-972.

-30 Kumar A, Sunita P, Jha S, Pattanayak SP: Daphnetin inhibits tnf-alpha and vegf-induced angiogenesis through inhibition of the ikks/ikappabalpha/nf-kappab, src/fak/erk1/2 and akt signalling pathways. Clin Exp Pharmacol Physiol 2016;43:939-950.

-31 Lee SH, Jung YS, Chung JY, Oh AY, Lee SJ, Choi DH, Jang SM, Jang KS, Paik SS, Ha NC, Park BJ: Novel tumor suppressive function of smad4 in serum starvation-induced cell death through pak1-puma pathway. Cell Death Dis 2011;2:e235.

-32 Zhang J, Wang J, Zhou YF, Ren XY, Lin MM, Zhang QQ Wang YH, Li X: Rich1 negatively regulates the epithelial cell cycle, proliferation and adhesion by cdc42/rac1-pak1-erk1/2 pathway. Cell Signal 2015;27:1703-1712.

33 Komaravolu RK, Adam C, Moonen JR, Harmsen MC, Goebeler M, Schmidt M: Erk5 inhibits endothelial migration via klf2-dependent down-regulation of pak1. Cardiovasc Res 2015;105:86-95.

-34 Huang M, Qiu Q, Xiao Y, Zeng S, Zhan M, Shi M, Zou Y, Ye Y, Liang L, Yang X, Xu H: Bet bromodomain suppression inhibits vegf-induced angiogenesis and vascular permeability by blocking vegfr2-mediated activation of pak1 and enos. Sci Rep 2016;6:23770.

-35 Naik MU, Naik UP: Contra-regulation of calcium- and integrin-binding protein 1-induced cell migration on fibronectin by pak1 and map kinase signaling. J Cell Biochem 2011;112:3289-3299.

-36 Gong Y, Yang X, He Q Gower L, Prudovsky I, Vary CP, Brooks PC, Friesel RE: Sprouty4 regulates endothelial cell migration via modulating integrin beta3 stability through c-src. Angiogenesis 2013;16:861-875.

-37 Makino Y, Tsuda M, Ohba Y, Nishihara H, Sawa H, Nagashima K, Tanaka S: Tyr724 phosphorylation of elmo1 by src is involved in cell spreading and migration via rac1 activation. Cell Commun Signal 2015;13:35.

-38 Schraufstatter IU, Trieu K, Zhao M, Rose DM, Terkeltaub RA, Burger M: Il-8-mediated cell migration in endothelial cells depends on cathepsin b activity and transactivation of the epidermal growth factor receptor. J Immunol 2003;171:6714-6722.

-39 Jia J, Ye T, Cui P, Hua Q, Zeng H, Zhao D: Ap-1 transcription factor mediates vegf-induced endothelial cell migration and proliferation. Microvasc Res 2016;105:103-108.

40 Huang X, Shen Y, Zhang Y, Wei L, Lai Y, Wu J, Liu X, Liu X: Rac1 mediates laminar shear stress-induced vascular endothelial cell migration. Cell Adh Migr 2013;7:462-468.

41 Caron C, DeGeer J, Fournier P, Duquette PM, Luangrath V, Ishii H, Karimzadeh F, Lamarche-Vane N, Royal I: Cdgap/arhgap31, a cdc42/rac1 gtpase regulator, is critical for vascular development and vegf-mediated angiogenesis. Sci Rep 2016;6:27485.

42 Zhang SC, Gremer L, Heise H, Janning P, Shymanets A, Cirstea IC, Krause E, Nurnberg B, Ahmadian MR: Liposome reconstitution and modulation of recombinant prenylated human rac1 by gefs, gdi1 and pak1. PLoS One 2014;9:e102425. 


\section{Cellular Physiology Cell Physiol Biochem 2017;41:1346-1359 \begin{tabular}{ll|l} 
DOI: 10.1159/000465389 & O 2017 The Author(s). Published by S. Karger AG, Basel \\
www.karger.com/cpb
\end{tabular} \\ Ju et al.: Autocrine VEGF and IL-8 Promote Migration of Endothelial Cells}

43 Garrett TA, Van Buul JD, Burridge K: Vegf-induced rac1 activation in endothelial cells is regulated by the guanine nucleotide exchange factor vav2. Exp Cell Res 2007;313:3285-3297.

44 Martin D, Galisteo R, Gutkind JS: Cxcl8/il8 stimulates vascular endothelial growth factor (vegf) expression and the autocrine activation of vegfr2 in endothelial cells by activating nfkappab through the cbm (carma3/bcl10/malt1) complex. J Biol Chem 2009;284:6038-6042.

45 Zhao LN, Wang P, Liu YH, Cai H, Ma J, Liu LB, Xi Z, Li ZQ, Liu XB, Xue YX: Mir-383 inhibits proliferation, migration and angiogenesis of glioma-exposed endothelial cells in vitro via vegf-mediated fak and src signaling pathways. Cell Signal 2017;30:142-153.

-46 Feng H, Hu B, Liu KW, Li Y, Lu X, Cheng T, Yiin JJ, Lu S, Keezer S, Fenton T, Furnari FB, Hamilton RL, Vuori K, Sarkaria JN, Nagane M, Nishikawa R, Cavenee WK, Cheng SY: Activation of rac1 by src-dependent phosphorylation of dock180(y1811) mediates pdgfralpha-stimulated glioma tumorigenesis in mice and humans. J Clin Invest 2011;121:4670-4684.

47 Zou L, Cao S, Kang N, Huebert RC, Shah VH: Fibronectin induces endothelial cell migration through beta1 integrin and src-dependent phosphorylation of fibroblast growth factor receptor-1 at tyrosines 653/654 and 766. J Biol Chem 2012;287:7190-7202.

48 Moshfegh Y, Bravo-Cordero JJ, Miskolci V, Condeelis J, Hodgson L: A trio-rac1-pak1 signalling axis drives invadopodia disassembly. Nat Cell Biol 2014;16:574-586. 